Research Article

\title{
Segmentation Algorithm for Intracranial Magnetic Resonance Images for Cerebral Stroke Identification and Nursing Evaluation
}

\author{
Wenjing Shi $\left(\mathbb{D},{ }^{1}\right.$ Xiangyu Kong $\left(\mathbb{D},{ }^{2}\right.$ Wei Tian $\mathbb{D D}^{3}$ Yujin Yan $\mathbb{D D}^{4},{ }^{4}$ and Yusi Chen $\mathbb{D D}^{2}$ \\ ${ }^{1}$ Department of Intensive Care Unit, Ningbo Municipal Hospital of TCM (Traditional Chinese Medicine), \\ Affiliated Hospital of Zhejiang Chinese Medical University, Ningbo 315000, Zhejiang, China \\ ${ }^{2}$ Department of Neurosurgery, Ningbo Municipal Hospital of TCM (Traditional Chinese Medicine), \\ Affiliated Hospital of Zhejiang Chinese Medical University, Ningbo 315000, Zhejiang, China \\ ${ }^{3}$ Department of Neurology, Ningbo Municipal Hospital of TCM (Traditional Chinese Medicine), \\ Affiliated Hospital of Zhejiang Chinese Medical University, Ningbo 315000, Zhejiang, China \\ ${ }^{4}$ Department of Neurosurgery, Affiliated Hospital of Ningbo College, Ningbo 315000, Zhejiang, China
}

Correspondence should be addressed to Yusi Chen; 201623201701011@zcmu.edu.cn

Received 14 March 2021; Revised 14 April 2021; Accepted 3 May 2021; Published 11 May 2021

Academic Editor: Gustavo Ramirez

Copyright (c) 2021 Wenjing Shi et al. This is an open access article distributed under the Creative Commons Attribution License, which permits unrestricted use, distribution, and reproduction in any medium, provided the original work is properly cited.

\begin{abstract}
This study aimed to investigate the therapeutic effect and nursing evaluation of patients with cerebral stroke (CS) through intracranial magnetic resonance imaging (MRI) images under the condition of segmentation algorithm. 199 CS patients were selected and divided randomly into a control group (group A) and an experimental group (group B) based on different treatment methods. Patients of group A were given routine antithrombotic therapy, and patients of group B were treated with intravenous thrombolytic therapy under evaluation of segmentation algorithm-based MRI images. Then, there were comparisons on clinical therapeutic effect, neurological damage score, and daily living ability index score of all patients. After treatment, the total effective rate $(92.12 \%)$ and daily life index $(41.45 \pm 11.24)$ of patients in group B were higher than those of group A $(P<0.05)$. However, neurological damage scores $(3.36 \pm 1.13$ points) of patients in group B after treatment were lower than those of group A $(5.85 \pm 2.31$ points $)(P<0.05)$. The routine clinical nursing satisfaction rate $(79.8 \%)$ was lower than the overall satisfaction rate (97\%) of the combination of clinical and imaging nursing $(P<0.05)$. Therefore, there were greatly clinical therapeutic effects of intravenous thrombolysis evaluated by intracranial MRI images under segmentation algorithm for CS patients, and routine nursing could improve patients' satisfaction, which were worthy of clinical promotion.
\end{abstract}

\section{Introduction}

Acute cerebral ischemic stroke (ACIS) refers to that there is cerebral artery blockage in patients and cerebral artery blood supply oxygen is insufficient suddenly, resulting in local brain tissue or cerebral nerve damage because of the obvious decrease in blood supply oxygen and the emerging of degenerative necrosis [1]. Patients often suffer from hemiplegia, hemiblindness, and partial sensory dysfunction due to ACIS. Besides, ACIS is a disease with high disability and fatality rates among diseases of the brain and blood circulation system. Its thrombolytic treatment has poor prognosis effect, which will cause a great degree of damage to the neural and physical functions of patients, and has seriously threatened the life and health of patients [2]. MRI is a kind of imaging examination for the internal organs of human body by using magnetic field and silent electric wave energy pulse, and the principle of nuclear magnetic resonance is applied to draw the image of internal structure [3].

Fuzzy clustering is a kind of algorithm often applied in segmentation of the CS image, in which fuzzy c-means (FCM) algorithm is more widely adopted [4]. Improper selection of the initial clustering center will have a huge impact on the accumulation results. Moreover, the FCM algorithm itself is complex in calculation, so it is difficult to use the spatial information of the image, resulting in 
complex image information and unclear edges. Studies have been pointed out that fast FCM algorithm can improve the computing speed. In order to deal with insufficient spatial information, the spatial FCM algorithm can be employed to segment the image, so as to make full use of the correlation between spatial information [5]. Clinically, plasminogen activator intravenous thrombolysis is often adopted to treat ACIS. However, the best time for intravenous thrombolysis is missed because MRI cannot accurately locate and diagnose ACIS, leading to the reduced therapeutic effect, various complications, and even intracranial hemorrhage, which is very unfavorable for the prognosis of patients [6]. With the continuous development of medical imaging technology, intracranial MRI image based on segmentation algorithm has gradually entered into clinical application, which not only provides reliable early diagnosis for ACIS, but also assesses the effective prognosis after thrombolytic therapy [7]. But there are few medical achievements in intracranial MRI image based on segmentation algorithm to evaluate the treatment of ACIS intravenous thrombolytic therapy. In this study, the clinical data of ACIS patients were taken as a reference, and MRI image was employed to assess the therapeutic effect and nursing of patients with CS. Highsensitivity C-reactive protein (hs-CRP) is one of C-reactive proteins. The ultrasensitive detection technology is applied by the clinical laboratory to accurately detect the low concentration of CRP, so as to enhance the sensitivity and accuracy of the experiment. In addition, hs-CRP level in the serum is closely related to the emerging, severity, and prognosis of acute cerebral infarction [8].

To sum up, the segmentation algorithm of FCM was applied to evaluate MRI images of patients with ACIS in order to assess the effect of intravenous thrombolytic therapy and investigate the satisfaction degree of patients under different ways of medical care, so as to provide reliable reference basis for clinic medicine.

\section{Materials and Methods}

2.1. Research Objects. 199 patients diagnosed as ACIS were selected as the research objects, who were admitted to hospital from September 10, 2019, to August 21, 2020, and their basic clinical data were collected. The Medical Ethics Committee of the hospital had approved this experiment and each patient and his/her family members had been informed of this experiment and signed the informed consent. Based on different treatment methods, the patients could be divided randomly into two groups, namely group A (43 females and 56 males with an average age of $62.56 \pm 4.65$ years and an average course of disease of $2.21 \pm 1.41$ hours) and group B (42 females and 58 males with an average age of $61.68 \pm 4.65$ years and an average course of disease of $2.37 \pm 1.25$ hours). There was no obvious difference in the data of patients from the two groups, which could be studied in groups.

The criteria for inclusion were defined to include patients who were up to the diagnostic criteria in Chinese Guidelines for the Diagnosis and Treatment of Acute Cerebral Ischemic
Stroke, suffered from ACIS for the first time, obtained 4-24 points of the National Institutes of Health Stroke Scale (NIHSS), and had the complete clinical data.

The criteria for exclusion were defined to include patients who suffered from posterior circulation CS combined with major diseases such as liver and kidney, had blurred MRI images, were over 70 years old, and dropped out of the experiment due to their own reasons.

\subsection{Hybrid Segmentation Algorithm of Multimode Magnetic} Resonance Imaging Two-Dimensional Images. FCM of the clustering algorithm was obtained by improving the hard clustering algorithm. The core purpose of the clustering algorithm was to find the appropriate clustering center and membership degree by solving the minimum variance and iteration variance of the clustering cost function. The value of the cost function was the weighted cumulative sum from the pixel to 2 norm measure of clustering center. Assuming that $X=\left\{x_{1}, x_{2}, x_{3}, \ldots, x_{n}\right\}$ was the eigenvalue of the image pixel, $A$ was the number of clustering centers from the divided $X$, the clustering centers represented $H=\left\{h_{1}, h_{2}, h_{3}\right.$, ..., $\left.h_{a}\right\}, g=\left\{g_{i k}\right\}$ was the membership matrix, and $g_{i k}$ stood for the membership degree of $x_{i}$ belonging to the region of $k$ th class. The cost function of FCM could be expressed in

$$
\operatorname{Min}_{m}(G, H)=\sum_{i=1}^{A} \sum_{j=1}^{n} g_{i j}^{m} b_{i j}^{2} .
$$

If the condition was satisfied, (2) could be obtained.

$$
\begin{aligned}
\sum_{i=1}^{A} g_{i j} & =1,1 \leq j \leq n, \\
\sum_{j=1}^{A} g_{i j} & >0,1 \leq i \leq a, \\
g_{i j} & =1 \leq i \leq a, 1 \leq j \leq n .
\end{aligned}
$$

In (2), $G=g_{i j}$ was an $n \times a$ fuzzy membership matrix, which represented the membership value of the $j^{\text {th }}$ sample $x_{j}$ belonging to the $i^{\text {th }}$ class, and the range was $0-1 . H=\left\{h_{1}, h_{2}\right.$, $\left.h_{3}, \ldots, h_{a}\right\}$ was the $s \times a$ matrix formed by $a$ cluster center vector.

In order to obtain the minimum value of the cost function $F(G, H)$, the Lagrangian multiplier method was adopted to construct the objective optimization function to calculate the partial derivatives of the objective function about clustering center $h_{i}$ and $g_{i j}$, so that the derivative result was 0 . Thus, the iterative expressions of the clustering center and membership degree were obtained as follows:

$$
h_{i}=\frac{\sum_{j=1}^{n}\left(g_{i j}\right)^{m} x_{j}}{\sum_{j=1}^{n} g_{i j}^{m}}, i=1,2, \ldots, a .
$$

Equation (3) stood for the partial derivative of the cluster center at $h_{i}, E_{j}=\{(i, j) x j=h j, \quad 1 \leq i \leq a\}$, and $g_{i j}$ could be expressed as (4) if $E j=\phi$. 


$$
g_{i j}=\left[\sum_{r=1}^{a} \frac{b_{i j}}{b_{r j}}\right]^{-1}, \quad i=1,2 \ldots a, j=1,2, \ldots n .
$$

The above equation represented the partial derivative of the cluster center at $g_{i j}$. If $E_{j}$ was nonzero, $g_{i j}$ was any nonnegative real number satisfying the following condition:

$$
\sum_{i=1}^{a} g_{i k}=1, g_{i k} \in[0,1] \text {. }
$$

The iterative equation of membership was actually a mapping from a point to a set. In the actual calculation process, the membership updating equation was often employed as

$$
\begin{aligned}
& g_{i j}=\left[\sum_{r=1}^{a} \frac{b_{i j} 2 / m-1}{b_{r j}}\right]^{-1}, \quad I_{j}=\phi, \\
& g_{i j}=\frac{1}{\left|I_{j}\right|}, \quad I_{j} \neq \phi, i \in I_{j}, \\
& g_{i j}=0, \quad I_{j} \neq \phi, i \notin I j .
\end{aligned}
$$

After sampling the original image, the sampled image was used as the input end of FCM to calculate the segmentation image.

2.3. Magnetic Resonance Imaging Scanning. Philips 3.0T MR Achieva (produced by General Electric Company, USA) was adopted in this experiment, and the whole process of examination was described to patients before scanning. They should keep breathing calm and were in the supine position during the examination, and the whole brain was scanned. The scanning parameters were as follows. The matrix was $251 \times 251$, thickness of layer was $3.5 \mathrm{~mm}$, field of view was $25 \times 25 \mathrm{~cm}$, flip angle was $15^{\circ}$, and layer spacing was $6.1 \mathrm{~mm}$. The scanning was officially started, T1-weighted imaging (T1WI) was set as spin recovery wave, the time to collect data twice was set, and the adequately collected data should be ensured. Flimpms was $448 \times 336$, the time to collect data four times was set, and flip angle was adjusted to $150^{\circ}$. After waiting for fluid attenuated inversion, flair would be recovered.

2.4. Therapeutic Methods. There were 99 subjects in group A and 100 subjects in group B. The patients of group A received routine thrombus therapy and were treated with platelet inhibitors and neuroprotective drugs. What is more, patients of group B were treated with intravenous thrombolytic therapy with plasminogen activator under the MRI image evaluation of segmentation algorithm. After the end of thrombolytic therapy for 24 hours, MRI images were applied to evaluate the diagnosis of reperfusion therapy, and aspirin was taken orally at $10 \mathrm{mg} /$ day. Patients of group A received MRI examination and routine hospital nursing services. The nursing staff paid close attention to the changes of patients' sign indexes in real time, patiently answered and timely dealt with the questions from patients and their family members, and appropriately pacified the patients' emotions. Patients in group B underwent MRI examination and were treated with the combination of imaging and clinical nursing on the basis of routine nursing services. Clinical nursing process had the following steps. First, the nursing staff should monitor the patient's vital signs in real time, observe the changes of patient's pupil, and immediately respond to the doctor once there were abnormal reactions. Second, the nursing staff needed to monitor and control the constant body temperature of the patient to reduce the damage degree of the patient's nerve functional tissue. Third, patients should be ensured that their breathing was smooth, and the nursing staff should pay attention to the changes of their vital signs at any time and appease the parents' emotions during the rescue. Imaging nursing process included 4 steps as follows. First, the nursing staff should fully understand the basic clinical data of the patient. The patient should not undergo MRI examination if it was found that he/she had a pacemaker or other equipment in the body. Second, the nursing staff should carefully check whether there were metal substances in the clothes of the patient before the MRI examination. If so, staff needed to help the patient to take off the clothes. Third, it was necessary to timely appease the emotions of patients and their family members and inform them of the matters needing attention in advance. Fourth, the nursing staff should focus on the changes of patients' vital signs in real time during the examination, inform the attending physician immediately if any abnormalities were found, and cooperate with the doctor to carry out the next treatment for patients after examination.

2.5. Observation Indexes. According to the diagnostic criteria of the Chinese Guidelines for the Diagnosis and Treatment of Acute Cerebral Ischemic Stroke, the cure rate was the reduction range of NIHSS score greater than or equaled to $90 \%$, the marked effective rate was NIHSS score reduction ranging from $46 \%$ to $89 \%$, the effective rate was the reduction range of NIHSS score for $18 \%-45 \%$, and the ineffective rate was NIHSS score reduction range less than or equaled to $17 \%$. Total effective rate $=($ marked effective rate + effective rate)/total number of cases $* 100 \%$. NIHSS was applied to evaluate the neurological function with a total score of 42 points. In addition, $0-1$ points represented the person was normal, 1-4 points stood for one with mild CS, 5-15 points expressed one with moderate CS, 16-20 points represented one with moderate and severe CS, and over 20 points meant one with severe CS.

The Barthel index was employed to evaluate the patient's daily living ability. The full score was 100 points, but the patient completely needed help in life if the score was less than 20 points. Patients with more than 20 points and less than 40 points needed great help in life. The scores were greater than 40 points and less than 60 points, the life of patients was in need of slight help.

Immune scattering turbidimetry was adopted to detect the hs-CRP level in serum. The emerging probability of complications was recorded in patients of the two groups. 
The adverse events were compared among patients in group $A$ and $B$. The satisfaction degree of patients and their family members was investigated by interview and questionnaire, and the results of satisfaction degree were statistically analyzed. Evaluation criteria were as follows. $0-7$ points meant they were dissatisfied, 7-8 points expressed they were basically satisfied, and 8-10 points revealed they were very satisfied.

2.6. Statistical Analysis. SPSS22.0 statistical software was employed to analyze and process the research data, and the calculation data of clinical therapeutic effect were expressed as the percentage (\%). Besides, the data information, in accordance with normal distribution, was expressed as mean \pm standard deviation $(-x \pm s)$. There were comparisons on NIHSS score, Barthel index, and CRP level in serum among the subjects from the two groups. $P<0.05$ meant that the results were statistically substantial.

\section{Results}

3.1. MRI Imaging Characteristics of Segmentation Algorithm in the Cerebral Stroke Patients. The manifestations of intracranial MRI imaging characteristics were as follows. Lacunar cerebral infarction was characterized by perforator arteriole occlusion, and predilection sites contained the basal ganglia region, hypothalamus, and brainstem, with a diameter of 10-15 mm and a long T1 and T2 signal (Figure 1). The normal null space signal disappeared and T1WI brain swelling was observed within 24 hours after the attack of ischemic stroke. When it was greater than 8 hours and less than 24 hours, T2-weighted imaging (T2WI) had high signal and $10-20 \%$ of it was negative (Figure 2).

\subsection{Comparison of Imaging Characteristics among Patients in} the Two Groups. The diffusion weighted imaging (DWI) signal characteristics of lesions were compared among patients in the two groups. There were $57.1 \%$ of patients in group A with mixed signals while $70.5 \%$ of patients in group $\mathrm{B}$ also showed mixed signals. Statistical data analysis was adopted, and the differences among patients in the two groups were statistically substantial $(P<0.05)$ (Figure 3$)$. There were high signal characteristics in $91.2 \%$ of patients of group A and $76.8 \%$ of patients of group B $(P<0.05)$ (Figure 4). DWI signal characteristics around lesions were compared among patients in the two groups. $73.2 \%$ and $83.5 \%$ of patients in the two groups presented high signal edema, respectively, showing a statistically significant difference (Figure 5).

\subsection{Comparison of Clinical Therapeutic Effects among Patients} in the Two Groups. According to the data, the cure, marked effective, and effective rates of patients in group B were higher than those of group $\mathrm{A}$, and the difference was statistically considerable $(P<0.05)$. The ineffective rate of patients in group $B$ was lower than the rate of group $A$ $(P<0.05)$. The total effective rate of patients in group $\mathrm{B}$ was greatly higher than that of group A $(P<0.05)$ (Figure 6). It indicated that the effect of intravenous thrombolysis was more marked than routine treatment under the condition of MRI image evaluation.

3.4. Comparison of the National Institutes of Health Stroke Scale Scores among Patients in the Two Groups. Before treatment, there was no huge difference in NIHSS score among patients in the two groups $(P>0.05)$. Based on different treatment methods, the NIHSS score $(3.36 \pm 1.13$ points) of patients in group B was sharply lower than that of group A (5.85 \pm 2.31 points), indicating that the difference was statistically remarkable $(P<0.05)$ (Figure 7$)$.

3.5. Comparison of Daily Living Ability Index Scores among Patients in Groups $A$ and B. The daily living ability index scores of patients in group A increased dramatically after treatment in contrast to group B, and the difference was statistically obvious $(P<0.05)$. The daily living ability index scores of patients in group $B$ grew markedly after treatment with a statistically significant difference $(P<0.05)$. Figure 8 shows that the daily living ability index scores of patients in group B $(41.45 \pm 11.24)$ after treatment were higher than those of group A $(32.93 \pm 8.41)$, with statistically great difference $(P<0.05)$. Therefore, it indicated that both routine treatment and intravenous thrombolysis under MRI imaging evaluation could reduce patients' nerve function damage and improve their daily living ability, while the latter was more effective and obvious.

3.6. Comparison of High-Sensitivity C-Reactive Protein in Serum among Patients in Groups $A$ and B. Before treatment, there was no huge difference of hs-CRP level in serum among patients from the two groups $(P>0.05)$. After treatment, hs-CRP level in serum of patients from group A reduced steeply, with statistically remarkable difference $(P<0.05)$. After treatment, the serum level of patients in group $\mathrm{B}$ was hugely lower than that of group $\mathrm{A}$, and the difference was statistically marked $(P<0.05)$ (Figure 9$)$. The results showed that the two treatments could reduce the hsCRP level in serum, and patients of group B had a more prominent effect.

3.7. Comparison of Adverse Reactions among Patients in the Two Groups. There were 3 patients with diarrhea, 2 patients with bleeding gums, and 4 patients with rash reaction in group A, with a total incidence of adverse reactions for $9.09 \%$. Besides, 3 patients of group B suffered from bleeding gums, 2 patients presented urethral bleeding, 2 patients had rash reaction, 5 patients suffered from phlebitis, and the incidence of adverse reactions was 12\%. Therefore, the difference was not statistically extreme among patients in the two groups $(P>0.05)$ (Figure 10).

3.8. Comparison of Nursing Satisfaction among Patients in the Two Groups. According to the results of the questionnaire survey, 50 patients and their family members of group A 


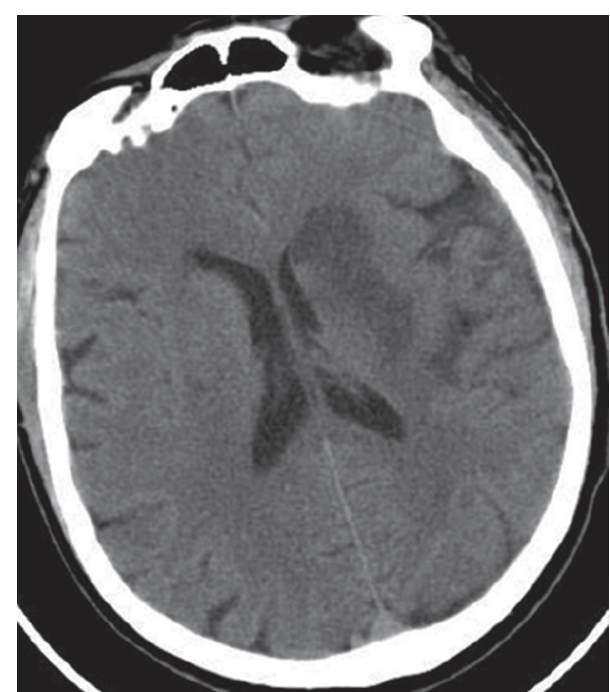

Figure 1: The image of lacunar cerebral infarction in the basal ganglia region.

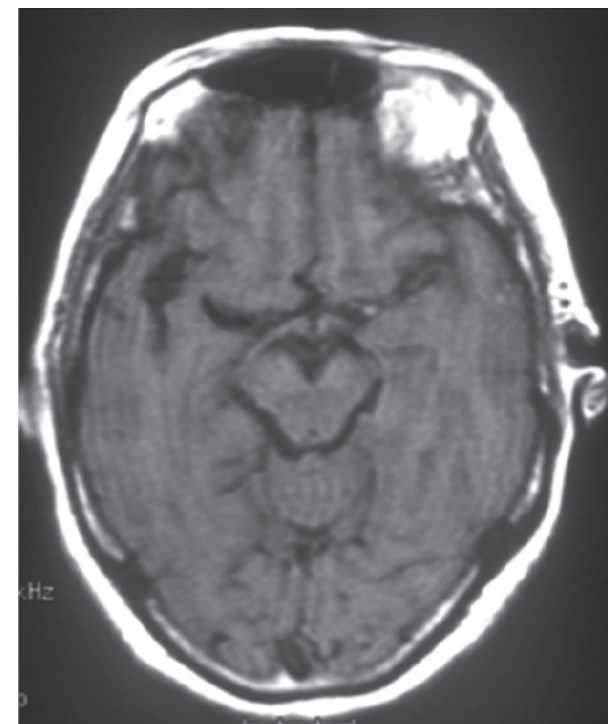

Figure 2: The image of patient's cerebral infarction for 4 hours.

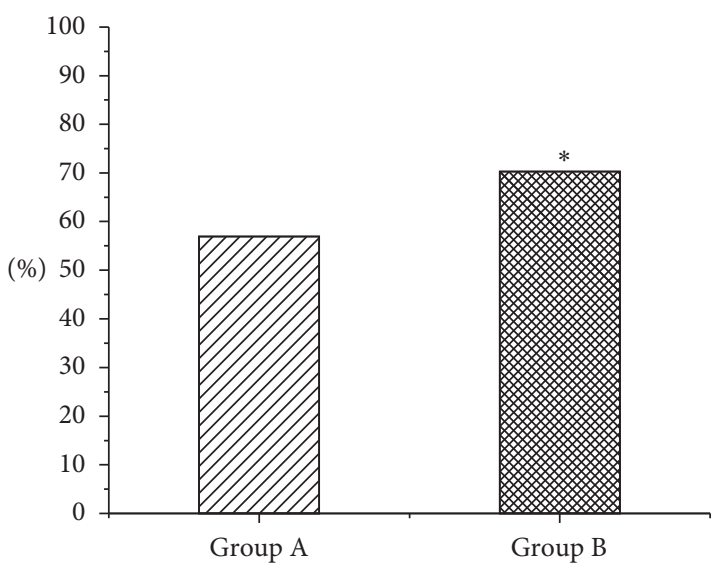

Figure 3: Comparison of mixed signals among patients in the two groups. (Note: * means there were statistically great differences by comparing with group A $(P<0.05))$.

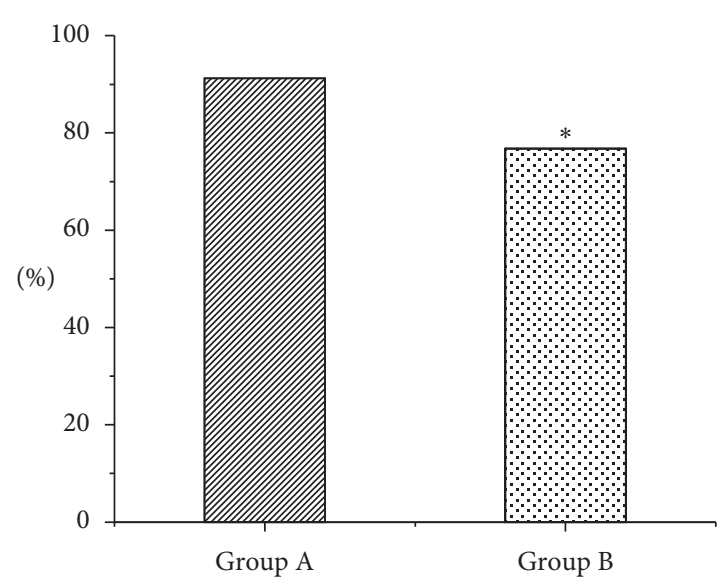

FIgURE 4: Comparison of high signals among patients in the two groups. (Note: * expresses there was a statistically great difference in contrast to group A $(P<0.05))$.

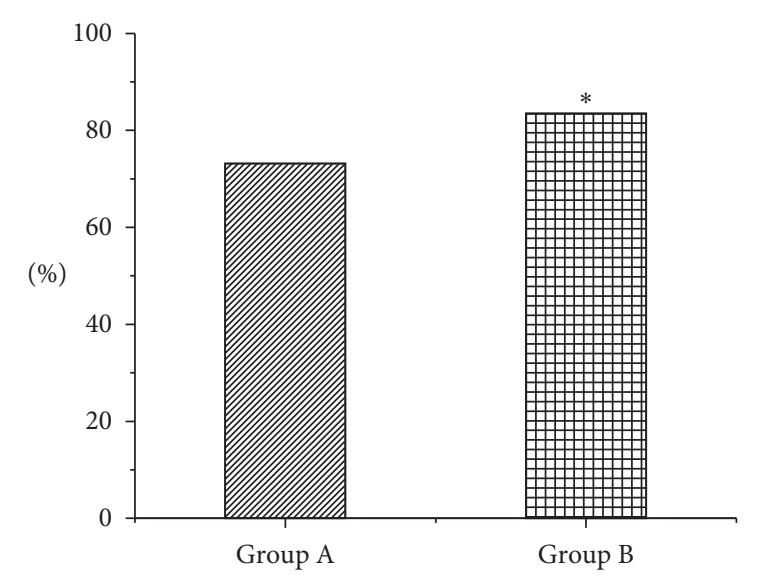

FIgURE 5: Comparison of high signal edema around lesions among patients in the two groups. (Note: *expresses the difference was statistically obvious compared with group A $(P<0.05))$.

were very satisfied, 33 were basically satisfied, and 16 were dissatisfied, with a total satisfaction rate of $79.8 \%$ (Figure 11). In group B, 74 patients were very satisfied, 23 were basically satisfied, and 3 were dissatisfied, with an overall satisfaction rate of $97 \%$ (Figure 12). There was a marked difference in nursing satisfaction among patients in the two groups, indicating that the statistical results were statistically substantial $(P<0.05)$.

\section{Discussion}

CS is caused by the inability of blood and oxygen to be normally delivered to the brain due to the obstruction or rupture of cerebral vessels and arteries, resulting in brain tissue damage or infarction, so that there is brain nerve function damage. Furthermore, CS has extremely high morbidity and mortality in cerebral blood diseases [9]. Clinically, MRI is commonly applied to examine ischemic stroke, and multimode MRI techniques include DWI, perfusion weighted imaging (PWI), magnetic resonance angiography (MRA), and other technical methods [10]. 


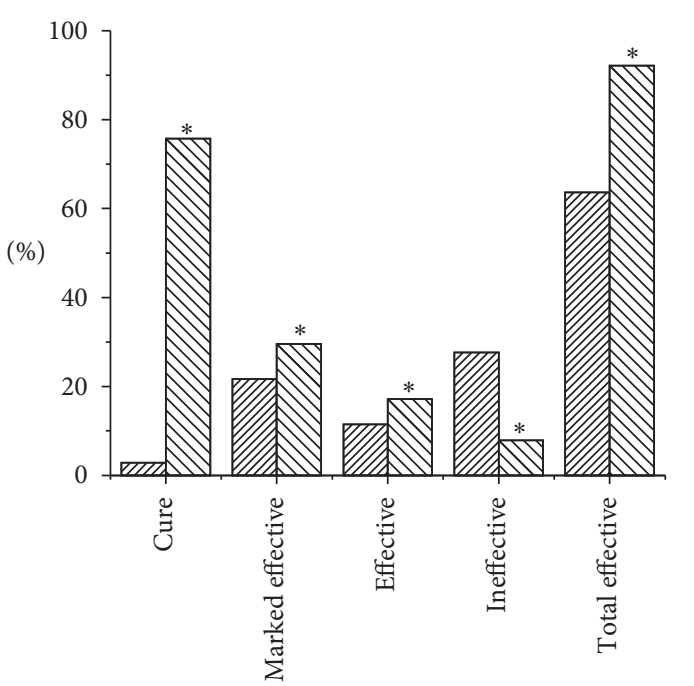

Group A

MV Group B

FIGURE 6: Comparison of clinical treatment effect among patients in the two groups. (Note: ${ }^{*}$ means the difference was statistically obvious in contrast to group A $(P<0.05))$.

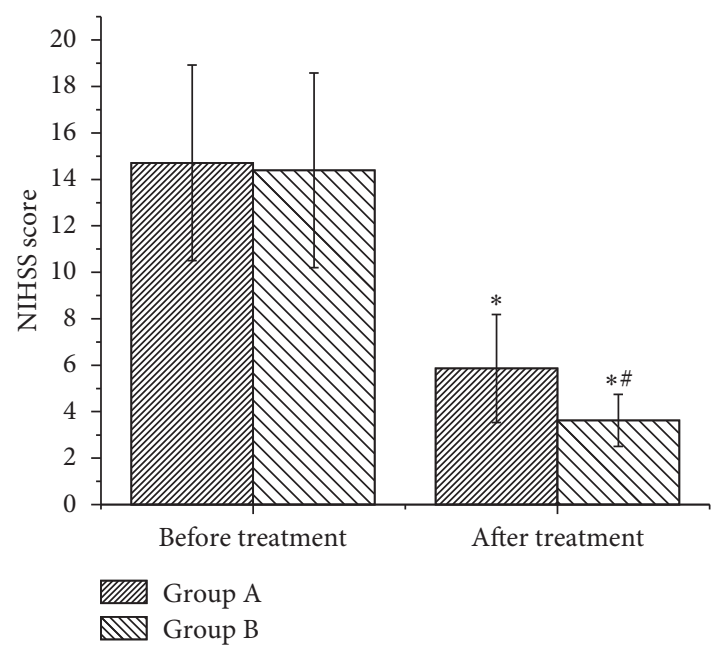

FIGURE 7: Comparison of NIHSS scores among patients in the two groups before and after treatment. (Note: ${ }^{*}$ shows that there was statistically significant difference compared with that of before treatment $(P<0.05)$, and \#indicates that the difference was statistically significant compared with group A after treatment $(P<0.05))$.

FCM can accelerate the calculation speed and make full use of spatial information correlation [11]. In the treatment of patients with ACIS, MRI image evaluation can accurately observe the signs of cerebral stem and cerebellar infarction, which is helpful for clinical diagnosis of patients with early CS [12]. Studies have reported that the sensitivity of MRI image evaluation is over $80 \%$ for the diagnosis of early CS [13]. Although MRI image evaluation has a good diagnostic effect and high safety performance for cerebellar infarction, small lesions, and basal ganglia removal during the examination of CS, its examination cost is also high. Compared

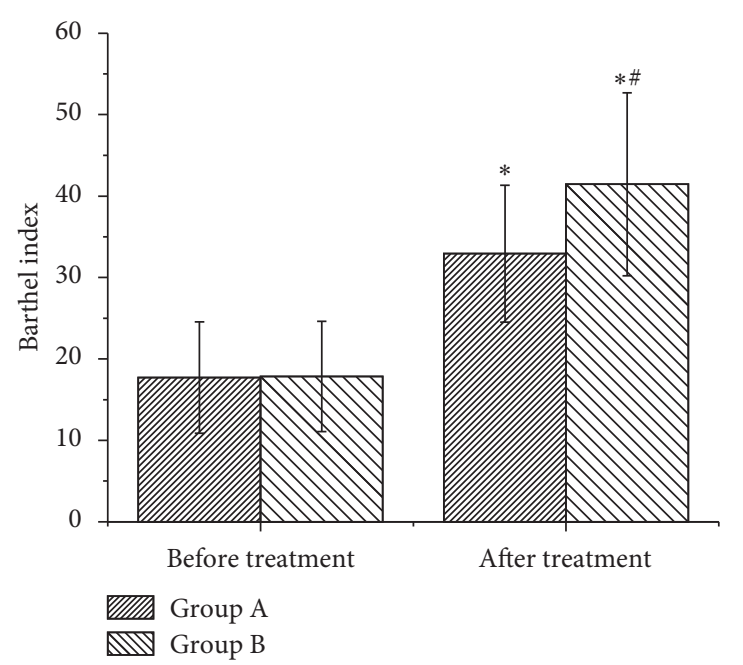

FIGURE 8: Comparison of daily living ability index scores among patients in the two groups. (Note: *means that there was statistically substantial difference compared to that of before treatment $(P<0.05$, and \#indicates that the difference was statistically significant in contrast to group A after treatment $(P<0.05))$.

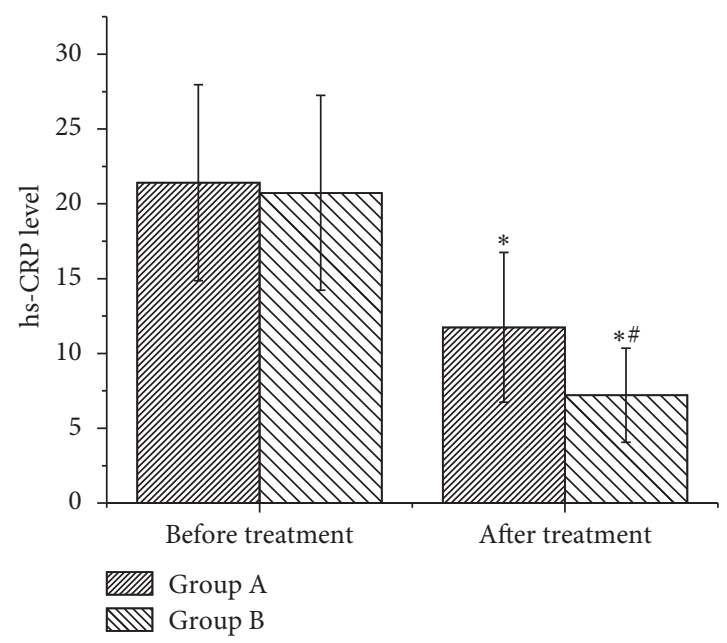

FIGURE 9: Comparison of hs-CRP levels in serum among patients in the two groups before and after treatment. (Note: ${ }^{*}$ means that there was statistically obvious difference in contrast to that of before treatment $(P<0.05)$, and \#reveales that the difference was statistically marked in contrast to group A after treatment $(P<0.05))$.

with popular consumption, patients are less likely to choose MRI image evaluation actively [14].

After treatment, the total effective rate (92.12\%), Barthel index scores $(41.45 \pm 11.24)$, and nursing satisfaction (79.8\%) of patients in group B increased markedly in contrast to group A. It was found that the clinical therapeutic effect of patients in group B after treatment was higher than the effect of group A, showing that intravenous thrombolysis treatment could obviously ease the patient's neurologic damage and promote the quality of life under the guidance of MRI. The hs-CRP level in serum and NIHSS score $(3.36 \pm 1.13$ points) of patients in group B were lower than 


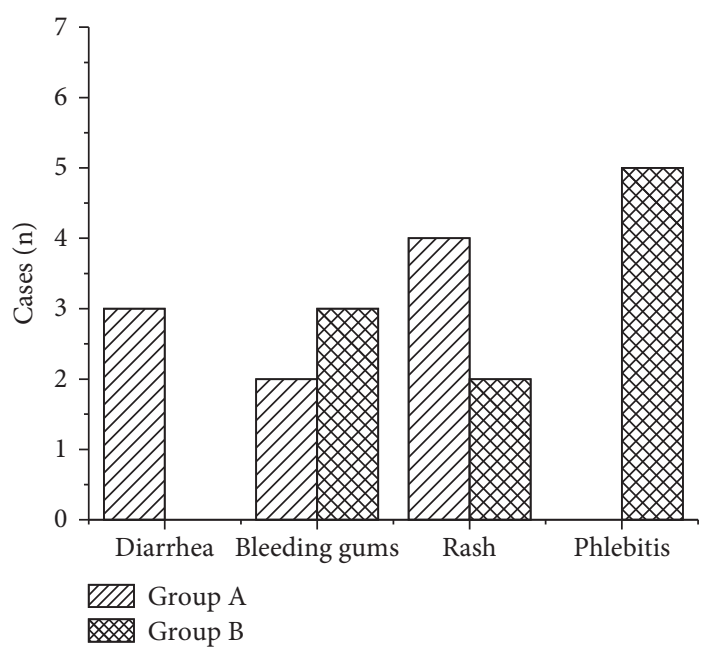

FIGURE 10: Comparison of adverse reactions among patients in the two groups.

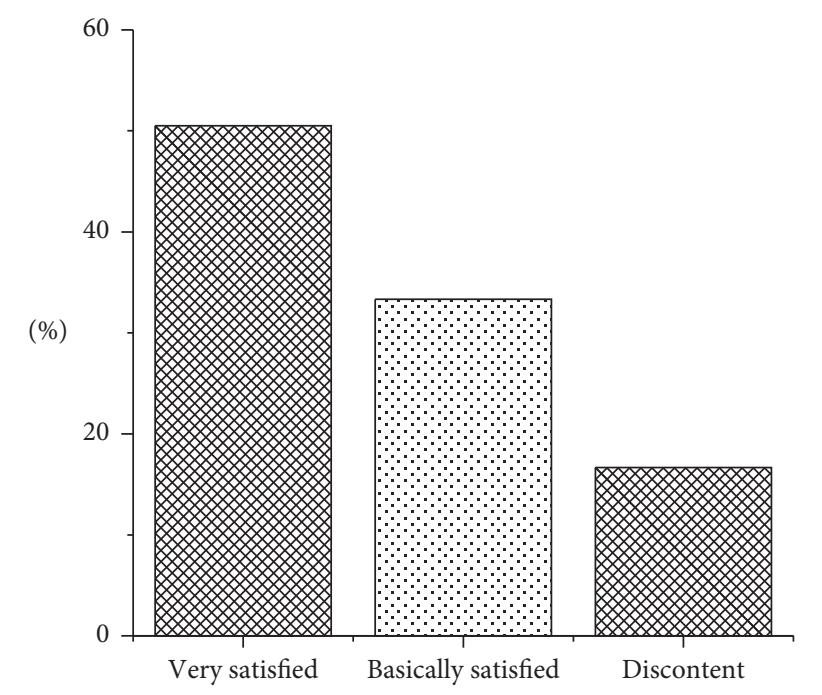

FIGURE 11: The nursing satisfaction results of patients in group A.

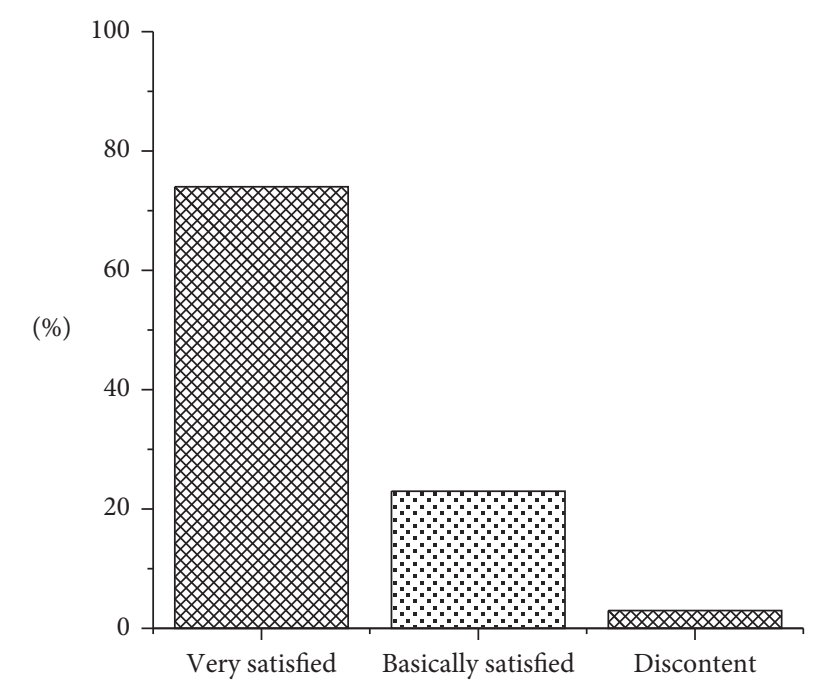

FIgURE 12: The nursing satisfaction results of patients in group B. 
those of group A, and there were no other complications, indicating that intravenous thrombolysis guided by MRI image evaluation was safe and helpful for patients' recovery. In the process of clinical MRI image evaluation, nursing staff should arrange reasonable nursing services, which was conducive to timely diagnosis and treatment of patients [15]. The combination of clinical nursing and image nursing was adopted in this study, which fully reflected the safety and efficiency. Clinical nursing can provide patients with the most basic vital sign change nursing, and the imaging nursing through MRI image evaluation can be in time based on professional nursing model; thus, combination of the two nursing methods can quickly assist patients to complete the examination projects, so as to reduce the examination time for diagnosis and treatment.

\section{Conclusion}

The intracranial MRI images based on segmentation algorithm were employed to evaluate intravenous thrombolysis in the treatment of ischemic stroke, which could effectively display the situation of cerebral vascular lesions. DWI could be applied to find high intensity signals, which could effectively reduce the neurological damage and improve their quality of life. The treatment results showed that there were no other complications, indicating that MRI imaging evaluation of intravenous thrombolytic therapy was safe and conducive to rapid recovery of patients. The combination of clinical and imaging nursing could dramatically enhance the nursing satisfaction of patients and their family members, which was better than routine nursing. Therefore, intracranial MRI images based on segmentation algorithm were assessing intravenous thrombolytic therapy, and a combination of clinical and imaging nursing could be further applied in clinical practice. The limitation of this study was that MRI image had a high cost of medical treatment, which was not applicable to patients with general consumption, and the research cost needed to be reduced in the later stage. In conclusion, the results of this study can provide a new diagnostic method for the treatment of patients with clinical CS.

\section{Data Availability}

No data were used to support this study.

\section{Conflicts of Interest}

The authors declare that they have no conflicts of interest.

\section{Acknowledgments}

This work was supported by Natural Science Foundation of Ningbo City, Zhejiang Province (2019A610364).

\section{References}

[1] J. J. Warner, R. A. Harrington, R. L. Sacco, and M. S. V. Elkind, "Guidelines for the early management of patients with acute ischemic stroke: 2019 update to the 2018 guidelines for the early management of acute ischemic stroke," Stroke, vol. 50, no. 12, pp. 3331-3332, 2019.

[2] K. Shibata, T. Hashimoto, T. Miyazaki, A. Miyazaki, and K. Nobe, "Thrombolytic therapy for acute ischemic stroke: past and future," Current Pharmaceutical Design, vol. 25, no. 3, pp. 242-250, 2019.

[3] J. D. Kaunitz, "Magnetic resonance imaging: the nuclear option," Digestive Diseases and Sciences, vol. 63, no. 5, pp. 1100-1101, 2018.

[4] V. S. Gurugubelli, Z. Li, H. Wang, and H. Fang, "eFCM: an enhanced fuzzy c-means algorithm for longitudinal intervention data," in Proceedings of the International Conference on Computing, Networking, and Communications, pp. 912916, Maui, HI, USA, March 2018.

[5] A. M. Anter and A. E. Hassenian, "CT liver tumor segmentation hybrid approach using neutrosophic sets, fast fuzzy c-means and adaptive watershed algorithm," Artificial Intelligence in Medicine, vol. 97, pp. 105-117, 2019.

[6] P. Ringleb, M. Bendszus, E. Bluhmki et al., "Extending the time window for intravenous thrombolysis in acute ischemic stroke using magnetic resonance imaging-based patient selection," International Journal of Stroke, vol. 14, no. 5, pp. 483-490, 2019.

[7] Y. Ran, Y. Wang, M. Zhu et al., "Higher plaque burden of middle cerebral artery is associated with recurrent ischemic stroke," Stroke, vol. 51, no. 2, pp. 659-662, 2020.

[8] Z. Cai, W. He, F.-J. Zhuang, and Y. Chen, "The role of high high-sensitivity C-reactive protein levels at admission on poor prognosis after acute ischemic stroke," International Journal of Neuroscience, vol. 129, no. 5, pp. 423-429, 2019.

[9] A. Knight-Greenfield, J. J. Q. Nario, and A. Gupta, "Causes of acute stroke," Radiologic Clinics of North America, vol. 57, no. 6, pp. 1093-1108, 2019.

[10] Q. Li, Z. H. Liu, H. Y. Wang et al., “Application of multimode MRI in prediction of nuclear grade of clear cell renal cell carcinoma," Zhonghua Yi Xue Za Zhi, vol. 99, no. 23, pp. 1767-1772, 2019.

[11] M. Ren, P. Liu, Z. Wang et al., "A self-adaptive fuzzy c-means algorithm for determining the optimal number of clusters," Comput Intell Neurosci, vol. 2016, Article ID 2647389, 12 pages, 2016.

[12] D. Mukherjee, V. Antar, B. Soylemez et al., "High-resolution diffusion tensor magnetic resonance imaging of the brainstem safe entry zones," Neurosurgical Review, vol. 43, no. 1, pp. 153-167, 2020.

[13] B. Manwani, S. Rath, N. S. Lee et al., "Early magnetic resonance imaging decreases hospital length of stay in patients with ischemic stroke," Journal of Stroke and Cerebrovascular Diseases, vol. 28, no. 2, pp. 425-429, 2019.

[14] Y. Iraha, M. Okada, R. Iraha et al., "CT and MR imaging of gynecologic emergencies," Radiographics, vol. 37, no. 5, pp. 1569-1586, 2017.

[15] I. Hong, A. Karmarkar, W. Chan et al., "Discharge patterns for ischemic and hemorrhagic stroke patients going from acute care hospitals to inpatient and skilled nursing rehabilitation," American Journal of Physical Medicine \& Rehabilitation, vol. 97, no. 9, pp. 636-645, 2018. 